\title{
Determinação de propriedades mecânicas do aço 1010 sob tensão no regime elástico por meio de speckle dinâmico
}

\section{RESUM 0}

Sidney Leal da Silva atec.professor.sidneyi@gmail.com Fatec Itaquera, São Paulo, São Paulo, Brasil.

Daniel José Toffol dantoffoli@gmail.com Fatec Itaquera, São Paulo, São Paulo, Brasil.

\section{Igor Catera de Biaso} igor 0071@hotmail.com Fatec Itaquera, São Paulo, São Paulo, Brasil.

Augusto Cesar Santos augustocesar.santos98@gmail.com Fatec Itaquera, São Paulo, São Paulo,

Charles Rodrigues da Silva charles.crdsr@gmail.com Fatec Itaquera, São Paulo, São Paulo, Brasil.

Henrique Trajano Araújo

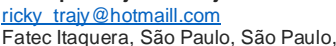
Brasil.
Utilizou-se a técnica de speckle aplicada a amostras de aço 1010 na busca de responder, conforme relata esse trabalho, se é possível relacionar os universos óptico e mecânico na determinação das propriedades dos materiais. A análise gráfica que compara os resultados de tensão versus deformação no regime elástico entre os universos óptico e mecânico mostrou que a relação entre os módulos de elasticidade é constante na amostragem utilizada. 0 valor médio encontrado para essa constante foi $\langle a\rangle=(7,9 \pm$ $0,6) \cdot 10^{-2}$ com uma diferença percentual relativa média entre as amostras de $1,0 \%$, indicando que esse valor é uma propriedade intrínseca do material. A diferença percentual média entre os coeficientes de Poisson ópticos e mecânicos foi 1,0\%, o que mostra que essa propriedade é igual nos dois universos estudados.

PALAVRAS-CHAVE: Elasticidade mecânica; Speckle; Interferência. 


\section{INTRODUÇÃO}

Diversas técnicas ópticas como speckle, holografia, fotoelasticidade etc., podem ser utilizadas para caracterização ou confirmação de determinadas propriedades físicas de materiais. Dentre essas técnicas, o speckle vem se destacando por possuir uma configuração experimental simples. Alguns trabalhos publicados mostram a ampla gama de aplicações destas técnicas, como em Engenharia Mecânica e de Materiais, em Física e em Medicina (KON e DINO, 2006; DA SILVA et al., 2016; SILVA e MURAM TSU, 2007).

Isaac Newton, em seu tratado Optiks, foi o primeiro a descrever qualitativamente o granulado óptico que surgia quando uma onda luminosa incidia em superfícies de materiais (NEWTON, 2015). Mais tarde, em um trabalho publicado em 1877, o pesquisador Exner descreveu a estrutura fibrosa vista quando a onda luminosa de uma lâmpada de mercúrio atravessava uma placa de vidro onde condensava vapor de sua respiração. Por volta de 1900, o pesquisador De Hass atribuiu a estrutura fibrosa à não monocromaticidade da radiação incidente. 0 formalismo matemático para o fenômeno inicialmente descrito por Newton foi introduzido por von Loue e lord Rayleigh na primeira década do século XX (ARIZAGA, TRIVI e RABAL, 1999).

A invenção do laser na década de 1960 proporcionou o avanço de técnicas, hoje conhecidas como speckle, que juntamente com seus diversos métodos tem sido largamente utilizada nos dias atuais para análise de superfícies de diversos materiais em diversas áreas do conhecimento, tanto científicas quanto tecnológicas, com a grande vantagem de não danificarem os materiais de estudo. Nesse sentido os ensaios por speckle podem ser considerados como não destrutivos (END).

Outra vantagem do speckle é a facilidade de montagem de sua configuração experimental. Com a computação, a dinâmica de análise dos dados se torna quase imediata e os resultados podem ser obtidos de maneira sistemática e com grande precisão.

No contexto descrito objetiva-se utilizar o speckle dinâmico para encontrar o módulo de elasticidade e o coeficiente de Poisson médio no aço 1010, sob tensão, no regime elástico, e comparar com os resultados obtidos por um processo não destrutivo tradicional da Engenharia Mecânica.

\section{TEORIA}

\section{SPECKLE E DEFORMAÇÃO ÓPTICA RELATIVA}

Speckle é um ramo da óptica que avalia o comportamento estático ou dinâmico do fenômeno de interferência em superfícies iluminadas por uma onda de luz coerente ao longo do tempo (SILVA e MURAMTSU, 2007). A Figura 1 mostra uma foto em tons de cinza da interferência formada durante a iluminação de uma gota de corretivo líquido, liquid paper, por um laser de Hélio-Neônio.

A ilustração mostra o resultado de um ruído no processo de formação da imagem, que é similar a um conjunto de grãos claros e escuros criando um efeito granulado, comumente conhecido como speckle (DA SILVA, 2017). 
Figura 1 - Interferência em 256 bits formada durante a iluminação de uma gota de corretivo líquido, liquid paper, por um laser de Hélio-Neônio

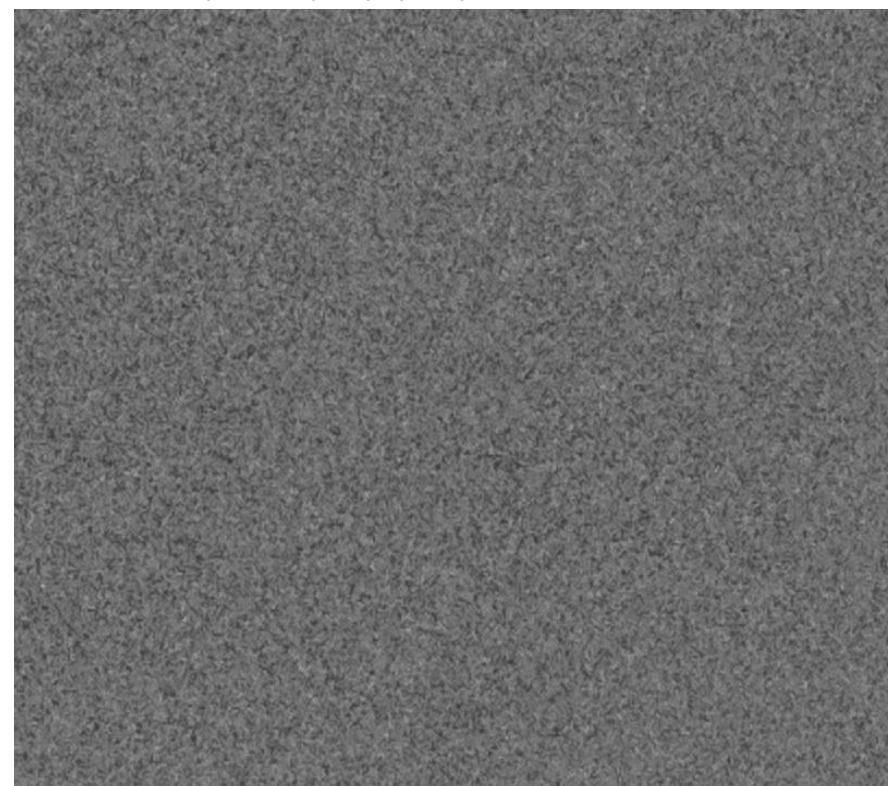

Fonte: DA SILVA (2017, p. 29)

A análise de conjunto de padrões instantâneos gera uma sequência de quadros que ao serem comparados com um padrão estático produzem os denominados interferogramas, como mostra a Figura 2.

Figura 2 - Interferograma a partir de dois padrões sucessivos de speckle na região de estudo da chapa de aço 1010.

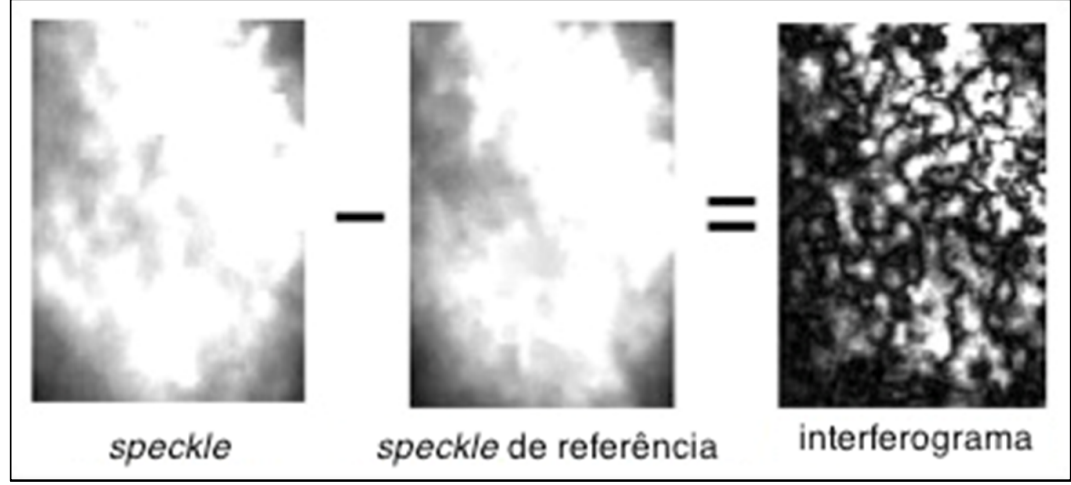

Fonte: Grupo de óptica e aplicações da Fatec Itaquera

A partir dos interferogramas é possível determinar as deformações ópticas relativas ao relacionar suas intensidades com a intensidade do speckle de referência, segundo a seguinte expressão (DA SILVA, 2017):

Deformação óptica relativa $\equiv \frac{\text { intensidade média (interferograma) }}{\text { intensidade média(referência) }}$ 
No processo óptico, o valor da deformação óptica relativa é a variável independente. Desta forma, a tensão será uma função dessa deformação, a menos de uma constante, que é a razão entre o módulo de elasticidade mecânico e a constante a que relaciona os universos óptico e mecânico (DA SILVA, 2017), tal que:

$$
\sigma_{\text {óptica }}=\frac{E}{a} \cdot \varepsilon_{\text {óptica }} \text {. }
$$

\section{ELASTICIDADE MECÂNICA}

Um corpo de prova, ao sofrer tensões que promovem alongamento apresenta a curva de tensão versus deformação mostrada na Figura 3.

Figura 3 - Relação tensão versus deformação na Elasticidade M ecânica

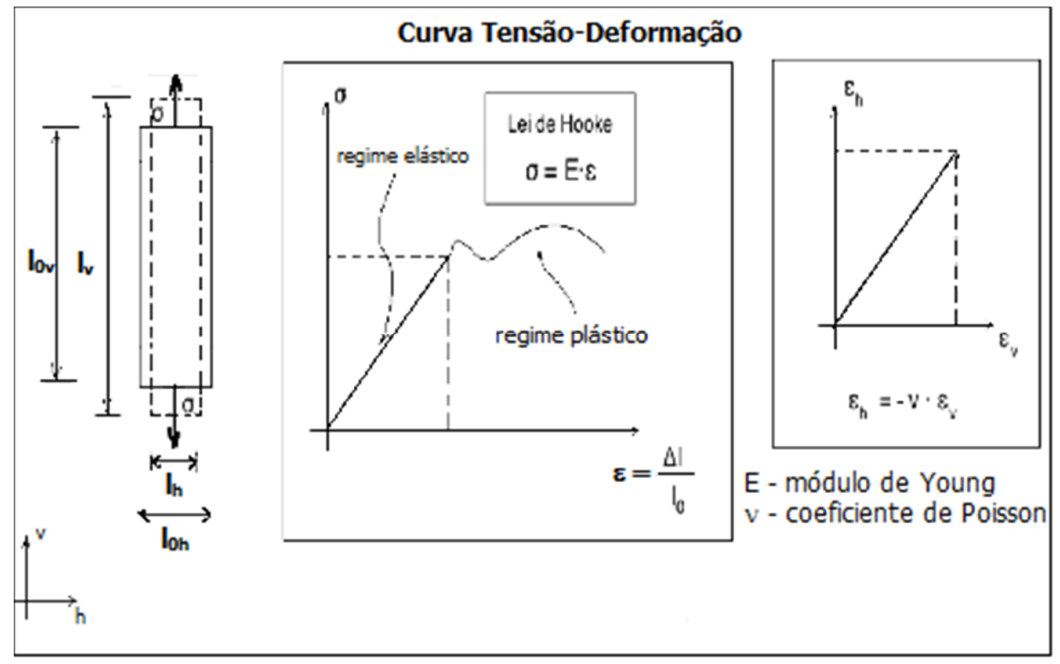

Fonte: Sidney Leal da Silva

Elasticidade Mecânica é o ramo da física que estuda as tensões e deformações de corpos materiais submetidos a esforços externos (FILHO, 2000). Tensão, $\rho$, é uma grandeza vetorial definida como a razão entre a força $\mathrm{F}$ e a superfície S de aplicação (NUSSENSZVEIG, 1998; SORIANO, 2003), tal que

$$
\vec{\rho}=\frac{\Delta \vec{F}}{\Delta S}
$$

Considerando a abordagem em módulo, sem perda em generalidade, a tensão axial ou normal, $\sigma$, é definida como

$$
\sigma=\frac{\mathrm{F}_{n}}{\mathrm{~A}}
$$

sendo $F_{n}$ o módulo da força resultante das forças axiais ou normais distribuídas uniformemente na seção de área $\mathrm{A}$. A dimensão da tensão é a dimensão de força por comprimento ao quadrado. No Sistema Internacional de unidades, a tensão é medida em $\left(\mathrm{N} / \mathrm{m}^{2}\right)$ ou Pa (Pascal).

Em 1676, Robert Hooke formulou que a tensão normal, $\sigma$, aplicada sobre um material linear é proporcional à sua deformação, a menos de uma constante de proporcionalidade, denominada módulo de elasticidade do material, E, ou módulo de Young (NUSSENSZVEIG, 1998; SORIANO, 2003). 
Thomas Young foi quem explicou essa formulação em 1807. A expressão, denominada Lei de Hooke, é dada por:

$$
\sigma=\mathrm{E} \cdot \varepsilon
$$

em que a deformação linear, $\varepsilon$, está relacionada ao comprimento original da barra e é definida por

$$
\varepsilon=\frac{\Delta l}{l}
$$

na qual $\Delta$ representa a variação de comprimento I da barra. $\varepsilon$, adimensional, é uma grandeza escalar já que tanto $\Delta \mathrm{l}$ quanto I, estão orientados na mesma direção. Uma barra tracionada deforma-se longitudinalmente, mas ao mesmo tempo apresenta uma contração lateral, que é uma fração da deformação longitudinal. Essa fração constante é denominada coeficiente de Poisson (v) e é dada por

$$
v=-\frac{\varepsilon_{\mathrm{p}}}{\varepsilon_{\mathrm{l}}} \Rightarrow \varepsilon_{\mathrm{p}}=-v \cdot \frac{\sigma}{\mathrm{E}}
$$

sendo $\varepsilon_{p}$ a deformação perpendicular e $\varepsilon_{l}$ a deformação longitudinal. 0 sinal (-) ajusta a expressão, já que geralmente as tensões positivas produzem deformações negativas e vice-versa.

\section{MATERIAIS, TÉCNICAS E MÉTODOS}

\section{AMOSTRAS}

Foram utilizadas quatro amostras de chapas de aço 1010 submetidas a tensões de tração progressivas aplicadas por uma máquina universal de ensaios da Time Group, no formato apresentado nas Figura 4 e 5.

Figura 4 - Chapa de aço 1010 acoplada à máquina de tração universal

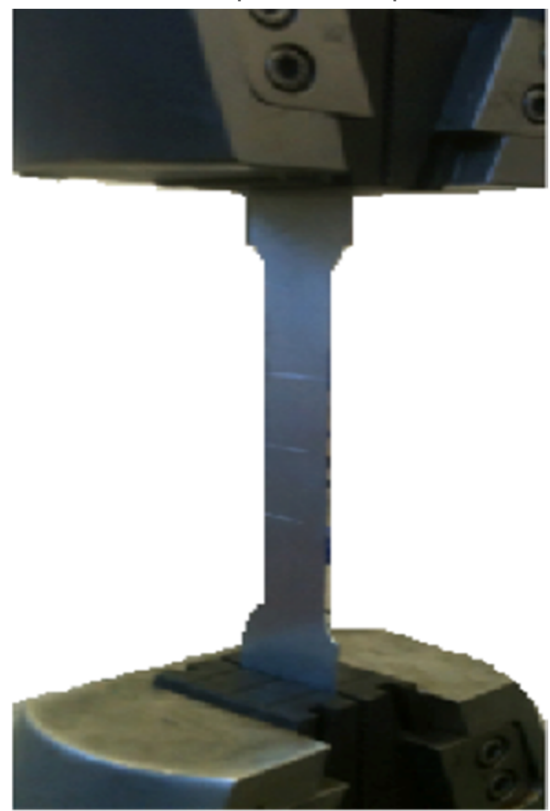


Figura 5 - Formato do corpo de prova em concordância com a DIN EN 10002-1

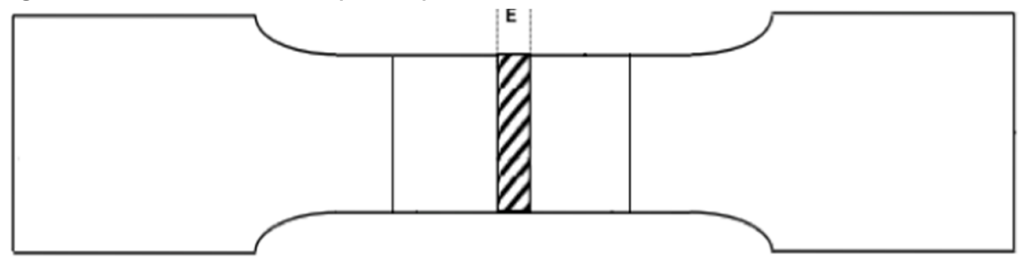

Fonte: Daniel José Toffoli

As dimensões das regiões centrais de estudo das chapas de aço (marcações no comprimento útil do corpo de prova, mostradas na Figura 5) são apresentadas no Quadro 1, obtidas por médias de três medidas utilizando o mesmo instrumento em cada amostra.

Quadro 1 - Dimensões das regiões de estudo nas amostras de chapas de aço

\begin{tabular}{|c|c|c|c|}
\hline Amostra & Comprimento $\left(10^{-2} \mathrm{~cm}\right)$ & Largura $\left(10^{-2} \mathrm{~cm}\right)$ & Espessura $\left(10^{-2} \mathrm{~cm}\right)$ \\
\hline 1 & $4,923 \pm 0,023$ & $2,159 \pm 0,010$ & $8,0000 \pm 0,032$ \\
\hline 2 & $4,938 \pm 0,027$ & $2,168 \pm 0,011$ & $7,9000 \pm 0,027$ \\
\hline 3 & $4,957 \pm 0,031$ & $2,158 \pm 0,009$ & $8,1000 \pm 0,039$ \\
\hline 4 & $4,892 \pm 0,015$ & $2,148 \pm 0,007$ & $8,1000 \pm 0,038$ \\
\hline
\end{tabular}

Fonte: Grupo de Óptica e Aplicações da Fatec Itaquera

\section{CONFIGURAÇÃO EXPERIMENTAL}

A Figura 6 apresenta um esquema da configuração experimental utilizada para captura dos dados no processo de speckle.

Figura 6 - Esquema da configuração experimental do speckle por reflexão

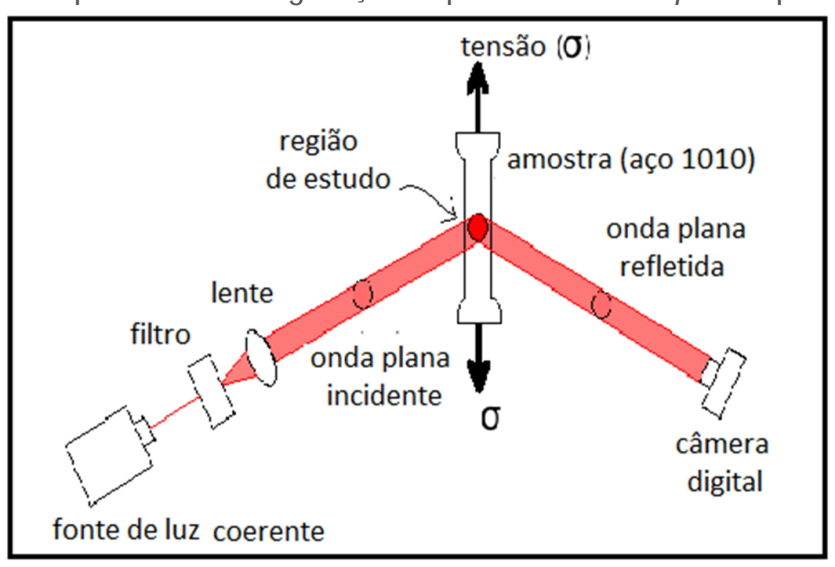

Fonte: Grupo de óptica e aplicações da Fatec Itaquera

Como fonte utilizou-se um laser com comprimento de onda $\lambda=632,8 \mathrm{~nm}$, faixa do vermelho, potência $5 \mathrm{~mW}$, que foi transformado em uma onda plana de intensidade aproximadamente constante, pelo uso de um filtro e uma lente.

A onda refletiu na amostra enquanto sofria esforços de tração, produzindo um padrão de interferências, formando o granulado dinâmico. Esse padrão foi registrado durante um determinado intervalo de tempo em uma câmera digital. 
Os vídeos foram utilizados como dados para a determinação dos interferogramas.

\section{PROCEDIMENTOS DE ANÁLISE}

Para determinar o padrão estático de speckle foi produzido um video com 4096 quadros da região de estudo iluminada pelo laser. Nessa situação, a amostra estava livre de esforços. A imagem foi convertida para 8 bits com 256 tons de cinza. A média entre as intensidades de todos os quadros produziu a imagem de referência.

Durante a aplicação contínua de tensão sobre a amostra, foi produzido um video com 12288 quadros. Cada quadro, em 8 bits, foi subtraído da imagem de referência, produzindo os interferogramas. Os cálculos das médias das intensidades dos interferogramas em relação à intensidade média da imagem de referência produziram as deformações ópticas relativas.

As relações entre os esforços, no regime elástico, obtidos diretamente da máquina universal de ensaios, e as deformações ópticas relativas, calculadas pela Expressão (1), geraram os gráficos de tensão versus deformação para o processo óptico. A obtenção do módulo de elasticidade foi obtida por método dos mínimos quadrados (VUOLO, 1992), a partir dos valores experimentais obtidos. Esses resultados foram comparados aos resultados obtidos a partir das curvas de tensão versus deformação do processo mecânico, levantadas automaticamente pelo sistema mecânico de tração. 0 processo foi repetido para outras três amostras do mesmo material.

\section{RESULTADOS E DISCUSSÕES}

As Figuras $\mathbf{7}$ e $\mathbf{8}$ apresentam os gráficos experimentais comparativos entre as tensões versus deformações obtidos nos dois processos, óptico (em azul) e mecânico (em preto) e, para as amostras 1 e 2, respectivamente, no regime elástico.

Figura 7 - Gráfico de tensão X deformação para a amostra 1

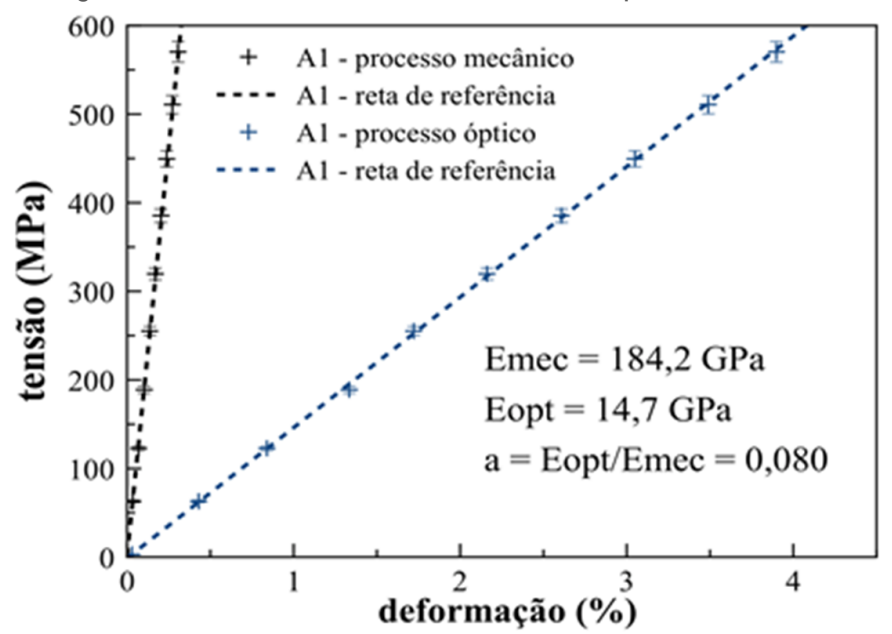

Fonte: Grupo de óptica e aplicações da Fatec Itaquera 
Figura 8 - Gráfico de tensão X deformação para a amostra 2

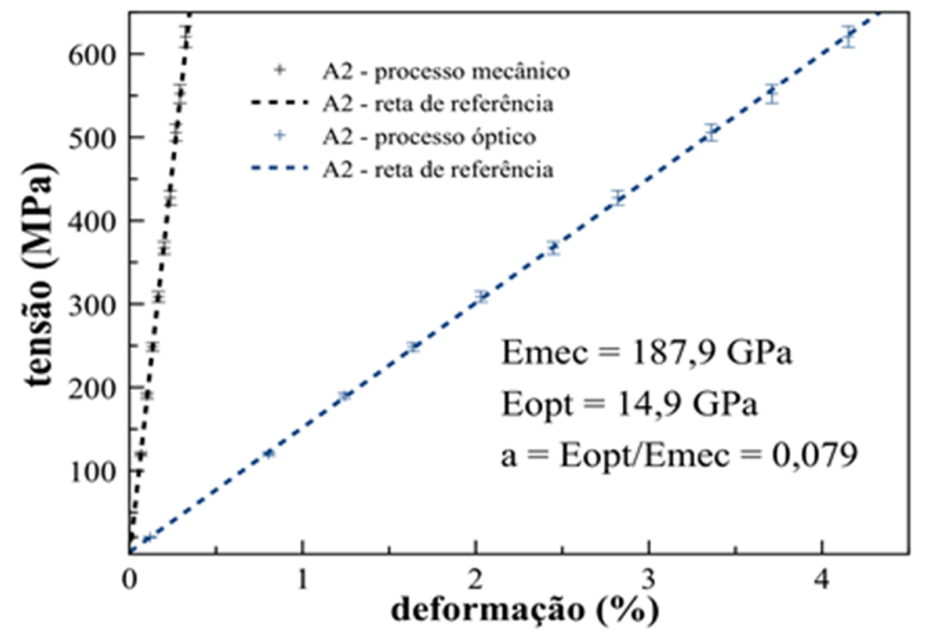

Fonte: Grupo de óptica e aplicações da Fatec Itaquera

No processo mecânico a variável independente é a deformação mecânica e a tensão (eixo vertical) é uma função dessa deformação pela Lei de Hooke. No processo óptico a variável independente é a deformação óptica, Expressões (1), e, nesse caso, a tensão (eixo vertical) é uma função dessa deformação, Expressões (2).

As barras de incertezas apresentadas nos gráficos já levam em consideração as incertezas do eixo horizontal, nos dois processos, pelo método dos mínimos quadrados (VUOLO, 1992). As relações entre as tensões e deformações são lineares em ambos os processos nas quatro amostras testadas.

A diferença no ângulo de incidência dos processos se dá pelo motivo de que no processo mecânico as tensões se relacionam diretamente com 0 deslocamento em cada ponto do material ao sofrer tração, já no processo óptico se relacionam com um padrão de intensidade obtido por meio do método proposto.

Os ajustes dos pontos experimentais, pelo método dos mínimos quadrados, forneceram os módulos de elasticidades em ambos os processos, cujos valores médios estão apresentados no Quadro 2.

Quadro 2 - Módulos de Elasticidade das amostras nos processos mecânico e óptico

\begin{tabular}{|c|c|c|}
\hline Amostra & $\begin{array}{c}\text { Módulo de elasticidade } \\
\text { mecânica }\left(10^{2} \mathrm{GPa}\right)\end{array}$ & $\begin{array}{c}\text { Módulo de elasticidade } \\
\text { óptica }\left(10^{2} \mathrm{GPa}\right)\end{array}$ \\
\hline 1 & $1,842 \pm 0,060$ & $1,471 \pm 0,046$ \\
\hline 2 & $1,879 \pm 0,059$ & $1,490 \pm 0,045$ \\
\hline 3 & $1,833 \pm 0,046$ & $1,450 \pm 0,040$ \\
\hline 4 & $1,916 \pm 0,055$ & $1,500 \pm 0,056$ \\
\hline
\end{tabular}

Fonte: Grupo de Óptica e Aplicações da Fatec Itaquera

Considerando as incertezas, tanto os módulos de elasticidade mecânicos quanto os módulos de elasticidade ópticos possuem valores iguais. Seus valores médios são apresentados no Quadro 3. 
Quadro 3 - Valores médios dos módulos de elasticidades

\begin{tabular}{|c|c|}
\hline Processo & Módulo de elasticidade (GPa) \\
\hline M ecânico & $(1,868 \pm 0,028) \cdot 10^{2}$ \\
\hline Óptico & $(1,478 \pm 0,024) \cdot 10^{2}$ \\
\hline
\end{tabular}

Fonte: Grupo de Óptica e Aplicações da Fatec Itaquera

A diferença percentual relativa média entre os valores dos módulos de elasticidades mecânicos é $2,2 \%$, enquanto que entre os valores dos módulos de elasticidades ópticos tal diferença é 1,7\%. As relações entre os módulos de elasticidade óptico e mecânico $(\boldsymbol{a})$ são apresentadas no Quadro 4.

Quadro 4 - Relações entre os módulos de elasticidades óptico e mecânico

\begin{tabular}{|c|c|}
\hline Amostras & $a\left(10^{-2}\right)$ \\
\hline 1 & $7,99 \pm 0,37$ \\
\hline 2 & $7,93 \pm 0,35$ \\
\hline 3 & $7,91 \pm 0,30$ \\
\hline 4 & $7,83 \pm 0,37$ \\
\hline
\end{tabular}

Fonte: Grupo de Óptica e Aplicações da Fatec Itaquera

Considerando as incertezas, os valores que relacionam os universos ópticos e mecânicos no regime elástico, para as quatro amostras utilizadas, são iguais. 0 valor médio é $\langle a\rangle=(7,91 \pm 0,17) \cdot 10^{-2}$. A diferença percentual relativa média entre os valores da constante <a> é $1,0 \%$. As Figuras 9 e 10 apresentam os gráficos experimentais comparativos entre as deformações perpendiculares aos esforços versus deformações longitudinais

Figura 9 - Gráficos experimentais das deformações perpendiculares x longitudinais para a amostra 1

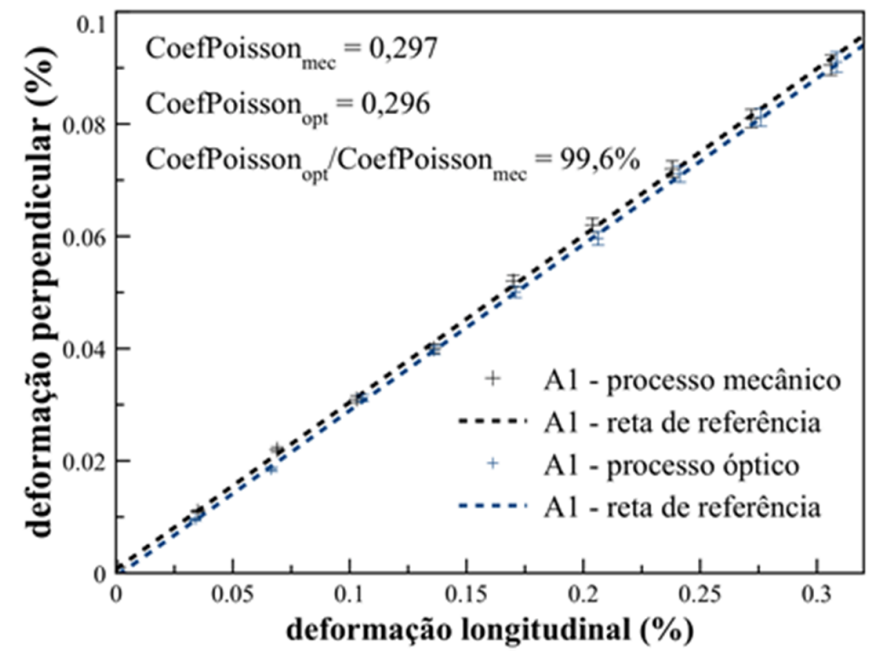

Fonte: Grupo de óptica e aplicações da Fatec Itaquera

As medidas das deformações perpendiculares aos esforços versus deformações longitudinais das Figuras 9 e 10, foram obtidas nos dois processos, óptico (azul) e mecânico (preto), para as amostras (1) e (2), respectivamente, no regime elástico. As deformações ópticas foram calibradas utilizando o coeficiente médio $<>$. 
Figura 10 - Gráficos experimentais das deformações perpendiculares x longitudinais para a amostra 2

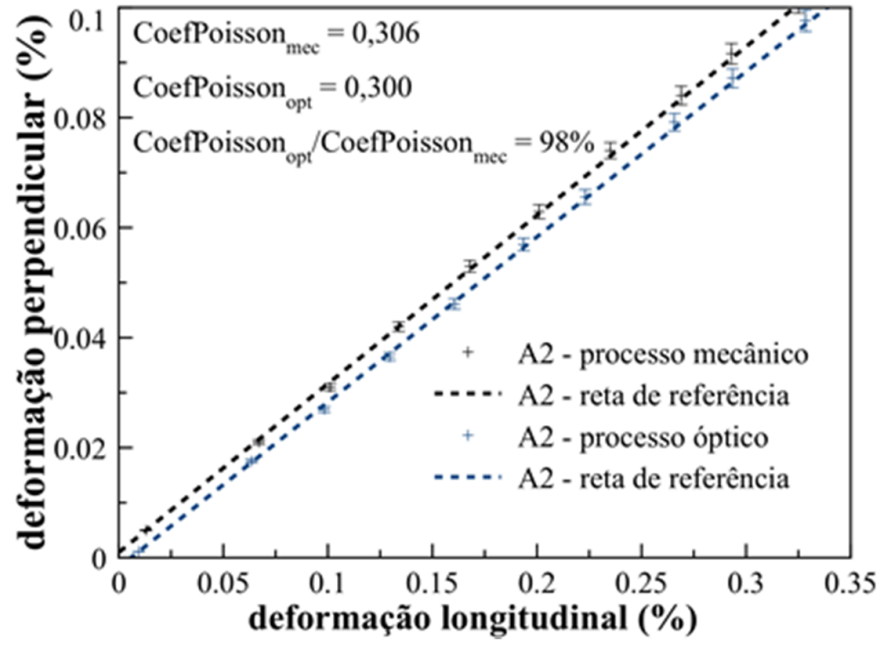

Fonte: Grupo de óptica e aplicações da Fatec Itaquera

As relações entre as deformações horizontais e verticais são lineares em ambos os processos nas quatro amostras testadas. Os ajustes dos pontos experimentais, pelo método dos mínimos quadrados, forneceram os coeficientes de Poisson em ambos os processos, cujos valores médios estão apresentados no Quadro 5.

Quadro 5 - Coeficientes de Poisson para as amostras nos processos mecânico e óptico

\begin{tabular}{|c|c|c|}
\hline Amostra & $\begin{array}{c}\text { Coeficiente de Poisson } \\
\text { mecânico }\left(10^{-1}\right)\end{array}$ & $\begin{array}{c}\text { Coeficiente de Poisson } \\
\text { óptico }\left(10^{-1}\right)\end{array}$ \\
\hline 1 & $2,97 \pm 0,12$ & $2,96 \pm 0,08$ \\
\hline 2 & $3,06 \pm 0,10$ & $3,00 \pm 0,09$ \\
\hline 3 & $3,01 \pm 0,13$ & $2,98 \pm 0,11$ \\
\hline 4 & $2,99 \pm 0,11$ & $3,01 \pm 0,10$ \\
\hline
\end{tabular}

Fonte: Grupo de óptica e aplicações da Fatec Itaquera

Considerando as incertezas, os coeficientes de Poisson mecânicos e ópticos possuem valores iguais. Seus valores médios são apresentados no Quadro 6.

Quadro 6 - Valores médios dos coeficientes de Poisson

\begin{tabular}{|c|c|}
\hline Processo & Coeficiente de Poisson $\left(10^{-1}\right)$ \\
\hline Mecânico & $3,01 \pm 0,23$ \\
\hline Óptico & $2,97 \pm 0,19$ \\
\hline
\end{tabular}

Fonte: Grupo de óptica e aplicações da Fatec Itaquera

A diferença percentual relativa média entre os valores dos coeficientes de Poisson na mecânica é $1,48 \%$, enquanto que entre os valores dos coeficientes de Poisson na óptica tal diferença é $0,83 \%$. A relação percentual média entre os coeficientes de Poisson ópticos e mecânicos é $99 \%$ e, portanto, são praticamente iguais nos dois universos.

Página | 10 


\section{CONCLUSÕES E PERSPECTIVAS}

As análises gráficas dos processos mecânicos forneceram valores médios compatíveis com a especificação do material, tanto para os módulos de elasticidade quanto para os coeficientes de Poisson.

Os gráficos obtidos pelo processo óptico tenderam ao mesmo comportamento dos gráficos obtidos no processo mecânico tradicional.

A relação entre os módulos de elasticidade, mecânicos e ópticos, mostrou a existência de um fator que associa o universo óptico ao universo mecânico, como uma propriedade intrínsica do material, já que seus valores se mostraram iguais para todas as amostras desse trabalho.

A relação entre os coeficientes de Poisson, mecânicos e ópticos também mostrou igualdade.

Os resultados ópticos apresentaram-se mais precisos em relação aos resultados mecânicos.

Esse trabalho mostrou que é possível utilizar o speckle dinâmico para determinar com precisão propriedades mecânicas do aço. No entanto, 0 aprimoramento da configuração experimental e a utilização de outras amostras de materiais dúcteis, permitirão melhorar os resultados.

No tratamento dos dados, outra possibilidade é tratar os interferogramas por meio da análise de Fourier (M OLTALVO et al., 2016). 


\title{
Determination of Mechanical Properties of 1010 Steel Under Tension in the Elastic Regime by Means of Dynamic Speckle
}

\begin{abstract}
In this work, the speckle technique has been applied to 1010 steel samples aiming to answer if it is possible to relate the optical and mechanical universes in order to determine the properties of the materials. The graphical analysis that compares the results of stress versus strain in the elastic regime, between the optical and mechanical universes, has shown that the relationship between the elastic moduli is constant in the sampling used. The average value found for this constant is $\left\langle a>=(7,9 \pm 0,6) \cdot 10^{-2}\right.$ with an average relative percentage differential between samples of $1.0 \%$, indicating that this value is an intrinsic property of the material. The average percentage difference between the optical and mechanical Poisson coefficientes is $1,0 \%$, showing that this property is equal in the two studied universes.
\end{abstract}

KEYWORDS: M echanical elasticity; Speckle; Interference. 


\title{
Determinación de las propiedades mecánicas del acero 1010 bajo tensión en la zona de deformación elástica por moteado dinámico
}

\begin{abstract}
RESUMEN
En este estudio, se utilizó la técnica speckle aplicada a muestras de acero 1010 con la intención de responder si es posible relacionar los universos óptico y mecánico en la determinación de las propiedades del material. El análisis gráfico que compara los resultados de la tensión frente a deformación en el rango elástico entre los universos óptico y mecánico ha mostrado que la relación entre los módulos de elasticidad es constante en la muestra utilizada. El valor medio para esta constante es $\varangle>=(7,9 \pm$ $0,6) \cdot 10^{-2}$ con un porcentaje de la diferencia relativa media entre las muestras de $1,0 \%$, lo que indica que este valor es una propiedad intrínseca del material. La diferencia media entre los coeficientes de Poisson óptico y mecánico fue 1,0\%, lo que demuestra que esa propiedad es la misma en los dos universos estudiados.
\end{abstract}

PALABRAS CLAVE: Elasticidad mecánica; M oteado; Interferencia. 


\section{AGRADECIMENTOS}

Os autores agradecem ao Grupo de óptica e aplicações, GOA, à Câmara de Ensino, Pesquisa e Extensão, CEPE, e à Direção da Fatec Itaquera pelo incentivo e apoio.

\section{REFERÊNCIAS}

ARIZAGA, R.; TRIVI, M.; RABAL, H. Speckle time evolution characterization by the co- occurrence matriz analysis. Optics \& Laser Technology - Elselvier, vol.31 p. 163(169), La Plata - Argentina, 1999.

DA SILVA, S. L.; JUNIOR, C. N.; JESUS, M. A.; VALSESHI, R.; DA SILVA, C. R.; AMORIM, T. C. A.; OLIVEIRA, S. Estudo do tempo de secagem em amostras líquidas com a técnica de Speckle. Revista Brasileira de Física Tecnológica Aplicada, v. 3, n. 2, p. 30-42, 2016. DOI: 10.3895/rbfta.v3n1.3676.

DA SILVA, S. L. Estudo de tensões em amostras fotoelásticas com Holografia Digital - Análise quantitativa. 1ํEdição: Novas Edições Acadêmicas, 2017, Brasil. $153 p$.

FILHO, A. A. Elementos Finitos, A Base da Tecnologia CAE. Primeira Edição. São Paulo: Editora Érica, 2000. 295 p.

KON; DINO, J. Estudo da evolução da reação de presa do cimento de fosfato de zinco através de luz laser, aplicando a técnica óptica de Speckle dinâmico. 2006. Dissertação (Mestrado em Odontologia) - Faculdade de Odontologia da Universidade de São Paulo.

M ONTALVO, R. A.; ASM AD, M; CHOQUE, I.; BALDWIN, G. Interferometria speckle para evaluar la flexíon de una barra de alumínio. Revista Mexicana de Física, v. 62 , p. 125-134, 2016.

NEWTON, I. Óptica. Tradução da 4. Edição de 1730 por André Koch Torres Assis. São Paulo: EDUSP, 2015. 296 p.

NUSSENSZVEIG, H. M. Curso de Física Básica: Fluidos, Oscilações e Ondas, Calor 4 ed., São Paulo: Editora Edgard Blüster Ltda, 1998.

SILVA, E.R.; MURAMATSU, M. O fenômeno do Speckle como introdução à metrologia óptica no laboratório didático, Revista Brasileira de Ensino de Física, vol. 29 n. 2, São Paulo, 2007. 
SORIANO, H. L. Método de Elementos Finitos em Análise de Estruturas, ed.1, São Paulo: Editora Edusp, 2003. 580 p.

VUOLO, J. H. Fundamentos da Teoria de Erros. São Paulo: Editora Edgard Blucher Ltda, 1992.

Recebido: 03 de fevereiro de 2017

Aprovado: 08 de abril de 2017.

DOI:

Como citar: SILVA, L.S. et al, Determinação de propriedades mecânicas do aço 1010 sob tensão no regime elástico por meio de speckle dinâmico, Revista Brasileira de Física Tecnológica Aplicada, Ponta Grossa,

v. 4 , n.1, p. $1-15$, mai./ jun. 2017

Contato: Sidney Leal Silva: fatec.professor.sidney@gmail.com

Direito autoral: Este artigo está licenciado sob os termos da Licença Creative Commons-Atribuição 4.0 Internacional.

(c) (9) 